\title{
Long-range cis-regulatory elements controlling CDF6 expression are essential for ear development
}

\author{
Guney Bademci, ${ }^{1}$ Clemer Abad, ${ }^{1}$ Filiz B. Cengiz, ${ }^{1}$ Serhat Seyhan, ${ }^{1}$ Armagan Incesulu, ${ }^{2}$ Shengru Guo, ${ }^{1}$ Suat Fitoz, ${ }^{3}$ Emine Ikbal Atli, ${ }^{4}$ \\ Nicholas C. Gosstola, ${ }^{1}$ Selma Demir, ${ }^{4}$ Brett M. Colbert, ${ }^{1,5}$ Gozde Cosar Seyhan, ${ }^{6}$ Claire J. Sineni, ${ }^{1}$ Duygu Duman, ${ }^{7}$ Hakan Gurkan, ${ }^{4}$ \\ Cynthia C. Morton, ${ }^{8,9,10,11,12}$ Derek M. Dykxhoorn, ${ }^{1,13}$ Katherina Walz, ${ }^{1,13}$ and Mustafa Tekin ${ }^{1,13,14}$
}

\begin{abstract}
John P. Hussman Institute for Human Genomics, University of Miami Miller School of Medicine, Miami, Florida, USA. Department of Otolaryngology, Eskisehir Osmangazi University School of Medicine, Eskisehir, Turkey. ${ }^{3}$ Department of Diagnostic Radiology, Ankara University School of Medicine, Ankara, Turkey. ${ }^{4}$ Department of Medical Genetics, Faculty of Medicine, Trakya University, Edirne, Turkey. ${ }^{5}$ Medical Scientist Training Program, University of Miami Miller School of Medicine, Miami, Florida, USA. ${ }^{6}$ Department of Dermatology, Bakirkoy Sadi Konuk Training and Research Hospital, Istanbul, Turkey.

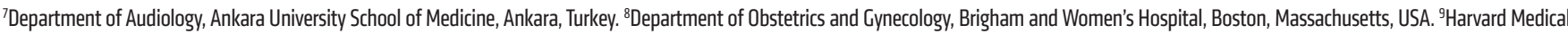
School, Boston, Massachusetts, USA. ${ }^{10}$ Program in Medical and Population Cenetics, Broad Institute of MIT and Harvard, Cambridge, Massachusetts, USA. "Department of Pathology, Brigham and Women's Hospital, Boston, Massachusetts, USA. ${ }^{2}$ Manchester Centre for Audiology and Deafness, School of Health Sciences, Faculty of Biology, Medicine and Health, Manchester Academic Health Science Centre, University of Manchester, Manchester, United Kingdom. ${ }^{3}$ John T. Macdonald Foundation Department of Human Genetics, and ${ }^{14}$ Department of Otolaryngology, University of Miami Miller School of Medicine, Miami, Florida, USA

Molecular mechanisms governing the development of the mammalian cochlea, the hearing organ, remain largely unknown. Through genome sequencing in 3 subjects from 2 families with nonsyndromic cochlear aplasia, we identified homozygous 221-kb and 338-kb deletions in a noncoding region on chromosome 8 with an approximately 200-kb overlapping section. Genomic location of the overlapping deleted region started from approximately 350 kb downstream of GDF6, which codes for growth and differentiation factor 6 . Otic lineage cells differentiated from induced pluripotent stem cells derived from an affected individual showed reduced expression of GDF6 compared with control cells. Knockout of Gdf6 in a mouse model resulted in cochlear aplasia, closely resembling the human phenotype. We conclude that CDF6 plays a necessary role in early cochlear development controlled by cis-regulatory elements located within an approximately 500 -kb region of the genome in humans and that its disruption leads to deafness due to cochlear aplasia.
\end{abstract}

\section{Introduction}

The inner ear is a complex organ that arises through a series of morphogenetic events from a simple embryologic structure, the otocyst. It consists of a dorsal vestibular system and a ventral auditory component that forms the cochlea. The cochlea houses hair cell auditory receptors, which translate sound into electrical signals deciphered by the brain $(1,2)$. Inner ear development requires a highly orchestrated process to establish otic identity in early development, including modeling of the structure with morphogenetic movements, expression of cell-specific proteins, and differentiation of the highly specialized cell types $(1,3)$. While model animals have been used to elucidate pathways and genes involved in these processes, the molecular mechanisms that govern early cochlear development in humans are poorly understood.

Hearing loss (HL) is the most common sensory deficit, affecting almost half of all people at some time in their lives. Clinically significant HL is present in approximately 1 per 500 newborns (4). Inner ear malformations, detected by computerized tomography or magnetic resonance imaging, are diagnosed in up to $25 \%$

Conflict of interest: The authors have declared that no conflict of interest exists. Copyright: () 2020, American Society for Clinical Investigation.

Submitted: February 3, 2020; Accepted: April 29, 2020; Published: June 29, 2020

Reference information: J Clin Invest. 2020;130(8):4213-4217.

https://doi.org/10.1172/JCl136951. of children with HL (5). One-third of these children have severe cochlear malformations associated with profound sensorineural HL (6). Cochlear malformations can be a phenotypic finding in a recognizable multisystemic condition, such as Pendred (OMIM 274600), branchiootorenal (OMIM 113650), or CHARGE (OMIM 214800) syndrome. The majority of cochlear malformations, however, are isolated, without additional phenotypic findings. DNA variants in very few genes have been reported to cause nonsyndromic HL associated with cochlear malformations (2, 7-10).

During embryonic development, tight regulation of gene expression in time and space is essential to shape a highly complex organism from a single cell. To achieve the required precision, core promoters close to transcription start sites must interact with noncoding regulatory elements in their vicinity, termed enhancers (11). Enhancers and promoters communicate across large genomic distances through direct physical contact via chromatin folding. A small number of noncoding DNA variants disrupting enhancers have been discovered in patients with congenital malformations (11). In this study, we aimed to determine molecular components of early cochlear development by undertaking a genetic study on congenital deafness associated with cochlear aplasia.

\section{Results and Discussion}

We studied 3 affected and 8 unaffected individuals from 2 Turkish families (Figure 1, A and B). The proband (individual IV:4 in Figure 
A

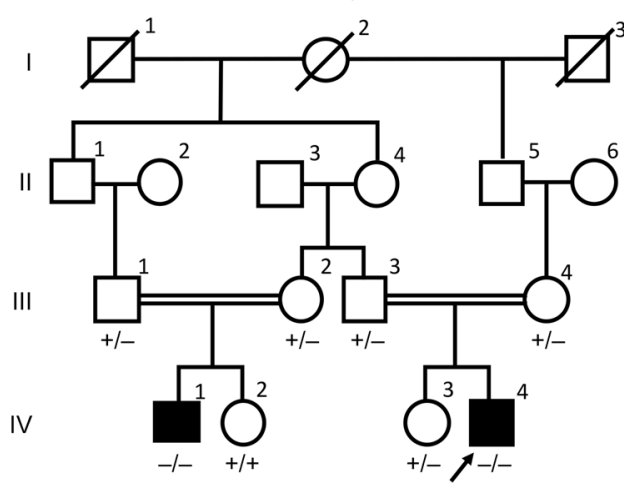

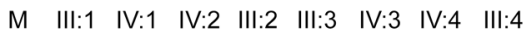

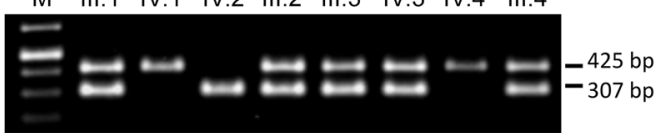

B
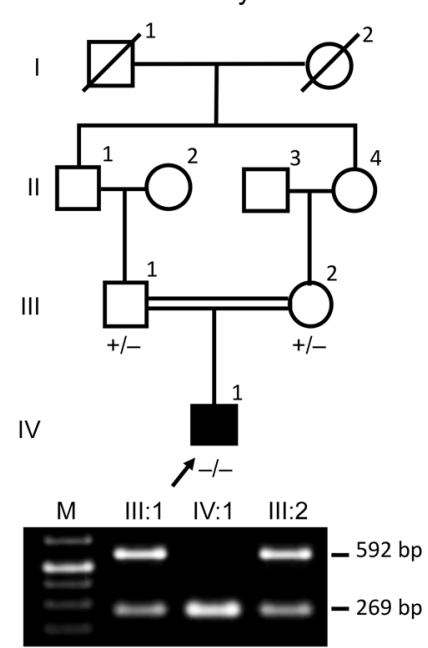

Figure 1. Clinical features and variant information. (A and $\mathbf{B}$ ) Pedigrees of the studied families with segregation of detected deletions. -/-, homozygous deletion; +/-, heterozygous deletion; +/+, homozygous WT; M: 1-kb ladder; amplicons of deletion-specific primers are 425 bp and 269 bp in families 1 and 2 , respectively; amplicons of WT-specific primers are $307 \mathrm{bp}$ and $592 \mathrm{bp}$ in families 1 and 2, respectively.

(C) Heavily T2-weighted MRI of temporal bone, axial maximum intensity projection, and volumerendered images when the proband of family 1 was 9 years old, showing complete absence of the cochlea. Vestibule and semicircular canals (blue arrows) are of normal shape and caliper. Control: MRI of a normal 9-year-old male reveals normal cochleas (red arrows) and semicircular canals.
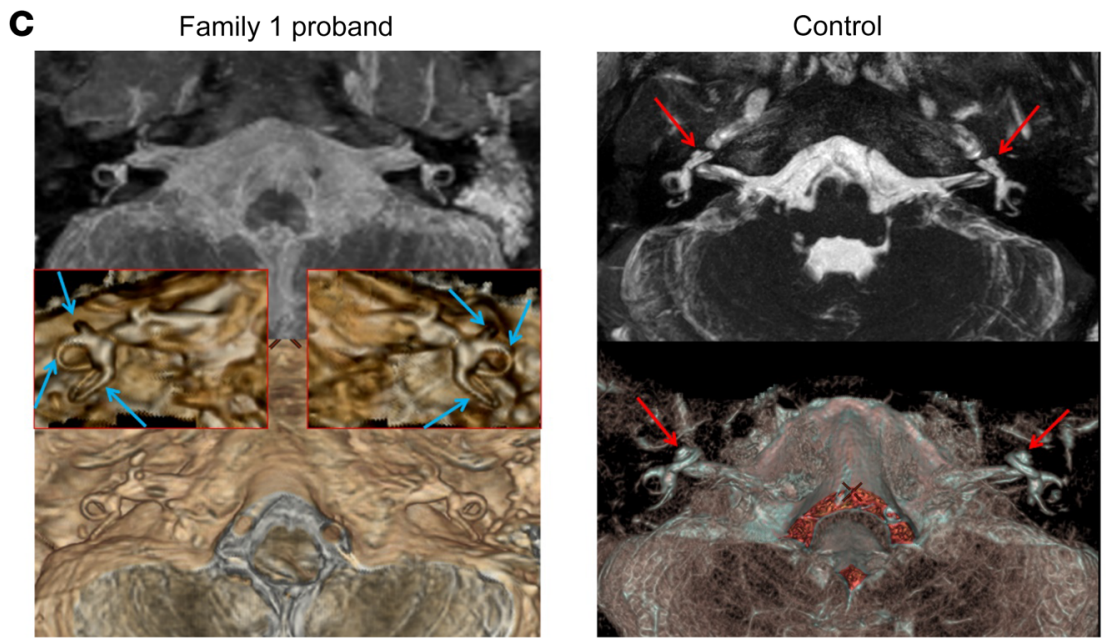

$1 \mathrm{~A}$ ) in family 1 is a male (20 years of age at the time of study) who was born with profound deafness to healthy consanguineous parents. His male cousin (20 years of age; IV:1 in Figure 1A) was born deaf as well. The proband in family 2 is a male (18 years of age; IV:1 in Figure 1B) who is also profoundly deaf. Pure tone audiograms in all 3 individuals showed profound sensorineural HL. Thorough clinical evaluations, including ophthalmological studies, have shown no additional phenotypic findings, suggesting nonsyndromic deafness. Specifically, these affected individuals did not have any skeletal abnormalities in the extremities or spine upon clinical examination, which was confirmed by x-rays. Development of gross motor skills was normal, and clinical examination showed normal tandem walking with a negative Romberg test, suggesting an intact vestibular system. Computerized tomography and magnetic resonance imaging of the temporal bone revealed cochlear aplasia in all 3 individuals, with normal-appearing vestibular systems (Figure 1C).

DNA samples from 2 affected individuals along with their normal-hearing siblings and parents in family 1 and from the proband in family 2 were subjected to genome sequencing. On average, read depth was $37.34 \times$; coverage at $1 \times$ and $10 \times$ was 99.37\% and 99.04\%, respectively (Supplemental Table 1; sup- plemental material available online with this article; https://doi. org/10.1172/JCI136951DS1).

We first evaluated the genomes of the 3 affected individuals separately for all known deafness genes (retrieved from Hereditary Hearing Loss Homepage, http://hereditaryhearingloss.org; and OMIM, https://www.omim.org) following recently published guidelines (12). This analysis did not reveal a plausible variant in any subject under any inheritance model. We subsequently evaluated single nucleotide variants (SNVs) and indels in the entire family 1 mapping to runs of homozygosity ( $>2 \mathrm{Mb})$ shared by both affected individuals (Supplemental Tables 2-4) and having an allele frequency of less than 0.0007 in the Genome Aggregation Database (global allele frequency; gnomAD, https:/gnomad. broadinstitute.org/) (12). From this list, we selected variants for which all 4 parents were heterozygous and the unaffected siblings were not homozygous for the variant allele. This analysis yielded 32 variants (Supplemental Table 5). None of these variants mapped to protein-coding regions; they were not located at conserved DNA sites (phastCons conservation $>0.4$ for vertebrates and mammals; http://compgen.cshl.edu/phast/phastConstutorial.php\#dnc); nor were they predicted to have an effect on gene function (Supplemental Table 5). We then checked segre- 
gating copy number variants (CNVs), which yielded only a single deletion, Chr8:96,582,049-96,803,788 (221,739 bp; hg19), in family 1 (Supplemental Table 6). This region was in the longest homozygous run $(5.4 \mathrm{Mb})$ shared by the 2 affected individuals in family 1 . The same locus was within an $18.2-\mathrm{Mb}$ homozygous run in the proband of family 2, who was homozygous for an overlapping deletion: Chr8:96,596,661-96,934,796 (338,135 bp; hg19). Sanger sequencing illuminated breakpoints of both deletions (Supplemental Figure 1). We confirmed the presence and segregation of both deletions, seq[GRCh37] $\operatorname{del}(8)(\mathrm{q} 22.1(96,582,048)$ : q22.1(96,803,789)) for family 1 and seq[GRCh37] del(8)(q22.1 (96,596,660):G:q22.1(96,934,797)) for family 2 using primer pairs specific for deletions via PCR (Figure 1, A and B, and Supplemental Table 7). These large deletions were not present in gnomAD, Database of Genomic Variants (http:/dgv.tcag.ca/dgv/app/ home), or DECIPHER (https://decipher.sanger.ac.uk/browser). Screening of each deletion in an additional 1025 unrelated Turkish individuals was negative, suggesting that these deletions are not common in the Turkish population. The single-locus 2-point lod score calculated using Superlink (13) with a disease allele frequency of 0.001 under a fully penetrant autosomal recessive model in the 2 families was 4.46 .

Topologically associating domain (TAD) analysis of the deleted region showed that both deletions were in the same TAD as GDF6 (codes for growth and differentiation factor 6; OMIM 601147) (Figure 2A). To demonstrate that GDF6 is involved in cochlear development, we undertook experiments to differentiate otic lineage cells from human iPSCs. To determine whether there were expression differences between affected and control individuals, we derived induced pluripotent stem cells (iPSCs) from fibroblasts of the proband in family 1 and of 2 age- and sexmatched controls. These affected and unaffected control iPSCs were differentiated into early otic lineage, nonneuronal ectoderm (NNE), and preplacodal ectoderm (PPE) cells, following an established stepwise differentiation protocol (Figure $2 \mathrm{~B}$ and ref. 14). We determined the expression of specific markers in the different stages with immunocytochemistry, RNA-Seq, and quantitative reverse transcriptase PCR (RT-PCR) (Figure 2B and Supplemental Figure 2).

RNA-Seq and RT-PCR revealed that GDF6 expression was absent on day 3 (iPSC) in both patient-specific and control cells. There was an increase in GDF6 expression on days 6 (NNE) and 11 (PPE) after initiation of differentiation in both the patient-specific and control cells (Figure 2B); GDF6 mRNA levels were significantly lower in the patient-specific compared with control cells on day 11 (Figure 2B and Supplemental Figure 3). Moreover, none of the 13 genes within $1 \mathrm{Mb}$ of the overlapping deletion region showed any significant differences in expression (Supplemental Figure 4). These results suggest that GDF6 expression begins during the early stages of inner ear development and that the deletion on chromosome 8 reduces its expression. Analysis of the deleted region showed multiple highly conserved DNA sequences and active chromatin positions, suggesting that this region contains enhancers for the regulation of gene expression (Figure 2A, Supplemental Figure 5, and refs. 15, 16).

Finally, to determine whether disruption of Gdf6 affects cochlear development, we evaluated a $G d f 6-\mathrm{KO}\left(G d f 6^{-/}\right)$ mouse model. Gdf6-KO mice do not survive postnatally and show fusion of the carpal and tarsal joints, coronal craniosynostosis, and middle ear defects (17). An inner ear phenotype in $G d f 6-K O$ mice has not been reported. First, we found that $G d f 6$ was highly expressed in the cochlea in both developing and adult WT mice (Supplemental Figure 6). We subsequently dissected and paint-filled $G d f 6$-mutant mouse inner ears to assess anomalies indicative of the human phenotype. Figure 2, C-E, shows defects in the mutant mice with cochlear aplasia, while the vestibular anatomy is normal.

The 207,127-bp deletion region on human chromosome 8 corresponds to $219,605 \mathrm{bp}$ of mouse chromosome 4 between 10,250,649 and 10,470,254 bp (Genome Reference Consortium GRCm38). Of these sequences, $11 \%$ are conserved in mammals and are identical in humans and mice (Supplemental Figure 7).

In this study we show that homozygous deletions removing putative enhancers of GDF6 lead to nonsyndromic HL associated with cochlear aplasia. GDF6 codes for growth and differentiation factor 6 , a member of the TGF- $\beta$ superfamily within the BMP family. It has been shown to play a role in normal formation of bones and joints in the limbs, skull, and axial skeleton (18). Heterozygous SNVs in GDF6 have been reported in multiple synostosis syndrome (OMIM 617898) (19); microphthalmia (OMIM 613094) (20); and Klippel-Feil syndrome (OMIM 118100), characterized by fused cervical vertebrae and a short neck, sometimes associated with sensorineural deafness (21). Three missense gain-of-function variants were shown to cause multiple synostosis syndrome by increasing the potency of GDF6 as a BMP signal $(19,22,23)$. A clear loss-of-function effect of the other reported amino acid substitutions has not been demonstrated, and their roles in monogenic congenital anomalies have been debated (22).

It has been proposed that expression of GDF6 is controlled by many tissue-specific enhancers that function during embryogenesis (24). Enhancers within an approximately 100-kb region flanking Gdf6 have been shown to control limb development (18). Deletions detected in our study map to an approximately $900-\mathrm{kb}$ region in the $3^{\prime}$ end of GDF6, which is a gene desert, a genomic region that is frequently associated with regulatory elements controlling expression of neighboring genes during embryonic development $(24,25)$. Multiple enhancers acting on the same promoter controlling differing spatiotemporal expression of the gene have been well described (11). Typically, enhancer-promoter interactions are complex due to the existence of multiple redundant enhancers with overlapping activities. A phenotypic manifestation in these cases can only be observed when variants disturb multiple enhancers (26). In rare instances, loss of a single enhancer may lead to a phenotype. For example, at least 11 enhancers driving the expression of $S h h$ in different tissues, such as central nervous system, epithelial lining, and limbs, have been reported (27). Removal of the limb-specific $Z R S$ enhancer eliminates $S h h$ expression only in the limbs, affecting limb outgrowth (27).

It has been recently shown that an approximately $123-\mathrm{kb}$ deletion within the mouse Slc25a13 gene on chromosome 5 is associated with reduced transcription of $D l \times 5$, which is located in cis at a distance of $660 \mathrm{~kb} . D l x 5$ is known to play role in inner ear development, and remarkably, Slc25a13-mutant mice showed inner 

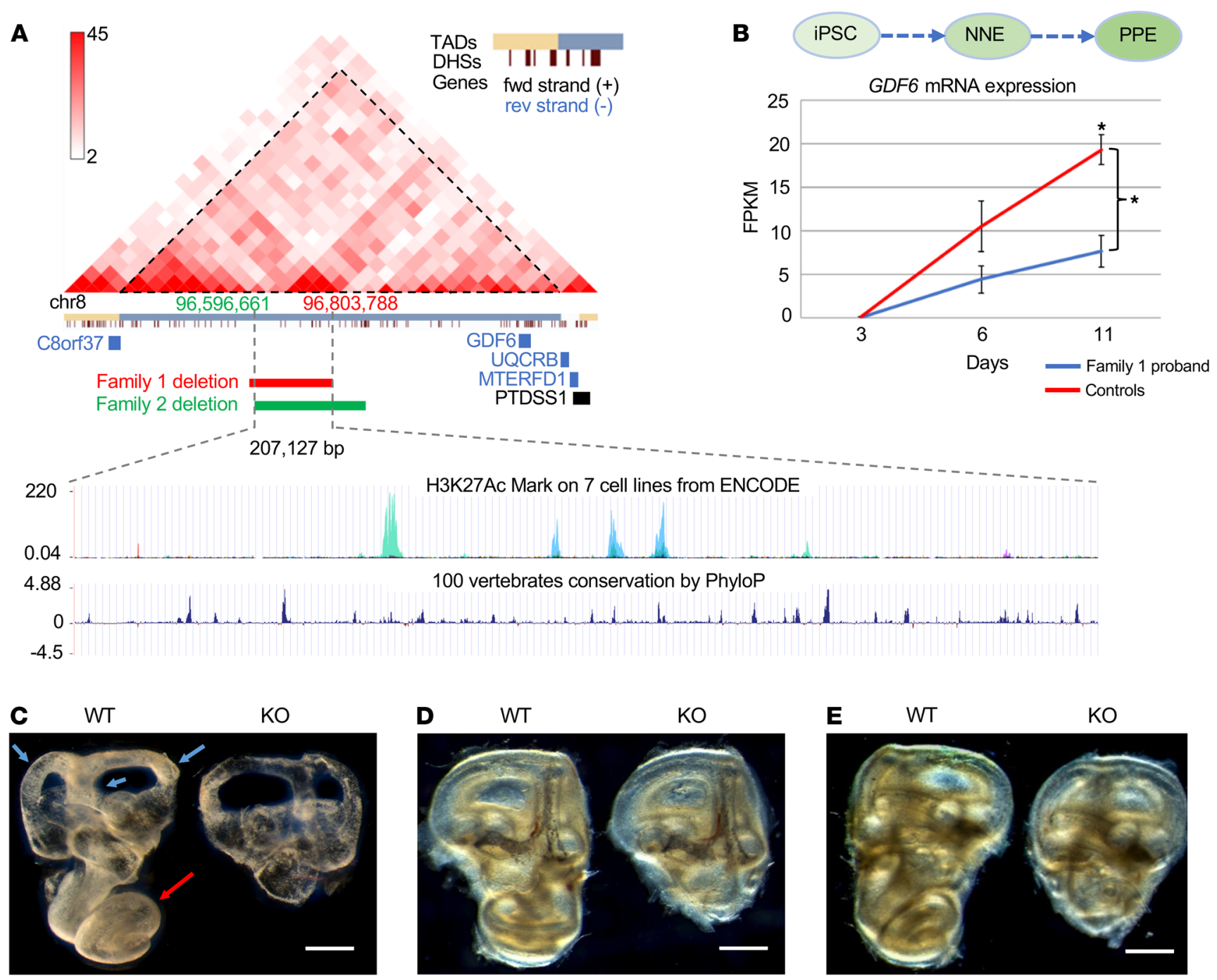

H3K27Ac Mark on 7 cell lines from ENCODE

100 vertebrates conservation by PhyloP

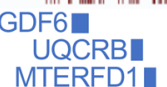

MTERFD1

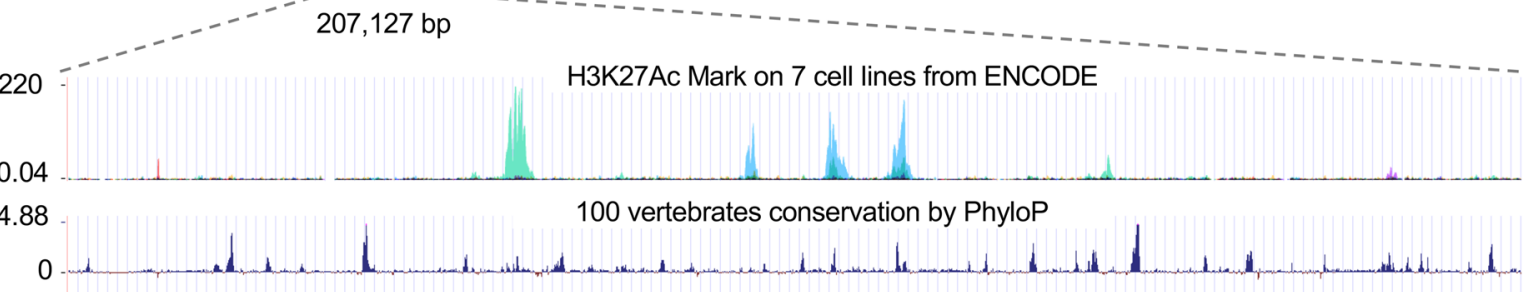

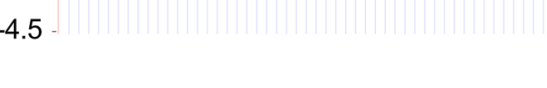
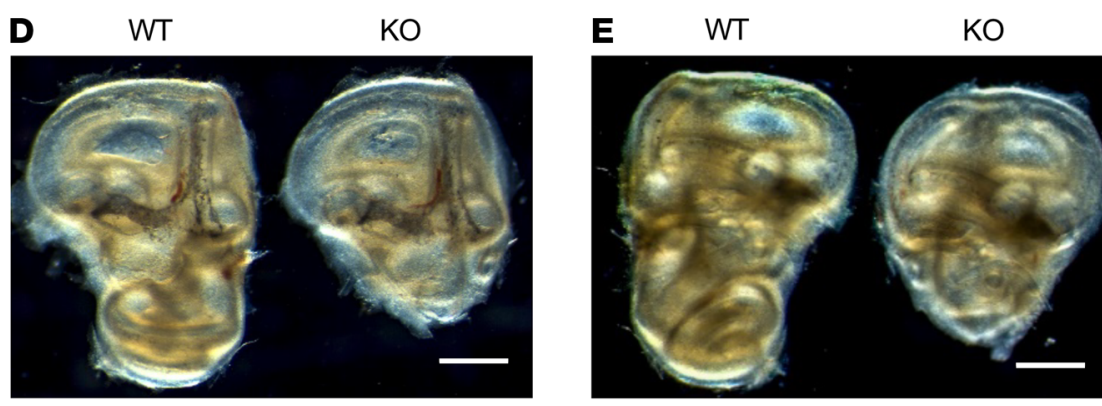

Figure 2. Deleted genomic region, mRNA expression, and mouse studies. (A) TAD on chromosome 8 including detected deletions (3D Genome Browser; http://promoter.bx.psu.edu/hi-c/chic.php). Boundaries of the TAD are shown with a blue bar under a triangle marked with dotted lines. The overlapping deletion is 207,127 bp shown with vertical dotted lines. This region contains multiple highly activated transcription sites, as demonstrated by the presence of the active enhancer mark H3K27Ac and DNAse I-hypersensitive sites (DHSs); the deleted region also includes multiple highly conserved DNA sequences, as shown by PhyloP (phylogenetic $P$ value) scores. ENCODE, Encyclopedia of DNA Elements. (B) GDF6 mRNA levels during differentiation from iPSCs. Data are represented as mean \pm SEM ( $n=4$ cases; $n=4$ controls). * For day 11 , mRNA levels and AUC between patient-derived and control cells were significantly different $(P=0.029)$. Mann-Whitney $U$ test with independent variables was used for comparisons. FPKM, fragments per kilobase of transcript per million mapped reads. (C-E) Cochlear aplasia in Gdf6-mutant mice. (C) Paint fill of PO mouse inner ear for WT and KO mice. The red arrow indicates normal cochlear morphology, and blue arrows show semicircular canals. ( $\mathbf{D}$ and $\mathbf{E})$ Bright-field photographs of the whole dissected inner ear on PO from different angles. Note that the Gdf6 mutant lacks the entire cochlea. Scale bars: $500 \mu \mathrm{m}$.

ear anomalies similar to those of $D l x 5^{-/-}$animals, reinforcing the concept that $S l c 25 a 13$ is a long-range Dlx 5 enhancer (28). Similarly, deletions within a $1-\mathrm{Mb}$ region upstream of POU3F4 on the $\mathrm{X}$ chromosome have been detected in patients with cochlear anomalies, suggesting the presence of cis-acting regulatory elements in the deleted regions (29).

The overlapping deletion region also includes the $3^{\prime}$ end of a long ncRNA (C8orf37-AS1). We did not detect RNA of this long noncoding RNA (ncRNA) in control iPSCs or in otic lineage cells. We also did not observe a difference in the expression of C8orf37 (Supplemental Figure 4). Further studies are needed to confirm the existence and characterization of this long ncRNA.
It is of interest that we observed only cochlear aplasia with an intact vestibule in the $G d f 6^{-/}$mice. While $G d f 6$ is expressed in both the cochlea and vestibule of developing and adult mice (Supplemental Figure 8 and ref. 30), its expression is more pronounced in the cochlea. It is possible that the lack of vestibular $G d f 6$ in $\mathrm{Gdf6}^{-/-}$mice is compensated by other genes. We propose that the overlapping deletions we detected in 3 patients leave GDF6 as well as other regulatory elements located outside of the deleted regions intact, and thus lead only to nonsyndromic deafness. It will be interesting to determine whether cochlear aplasia is due to the deletion of only one enhancer or the combined effects of multiple enhancers mapping to the deleted region. 


\section{Methods}

Complete details of the experimental materials and methods are provided in Supplemental Methods.

Genome and RNA sequencing data were deposited in the NCBI's BioProject database: PRJNA623118, BioSample: SAMN14539183 and SAMN14539184; and PRJNA626451, respectively.

Statistics. Differences between mRNA levels of patient and control samples were compared with nonparametric test (Mann-Whitney $U$ test with independent variables). A $P$ value less than 0.05 was considered significant.

Study approval. This study was approved by the Ethics Committee of Ankara University in Turkey (no. 012413) and the Institutional Review Board of the University of Miami (no. 20081138). A signed informed consent form was obtained from each participant or, in the case of a minor, from parents. All procedures were approved by the University of Miami Institutional Animal Care and Use Committee and followed the NIH guidelines "Using Animals in Intramural Research.”

\section{Author contributions}

MT designed the study. AI, SD, DD, HG, SS, GCS, EIA, SF, and MT performed clinical evaluation and collected samples. GB, CA, FBC, SS, SG, NCG, BMC, and CJS developed methods and analyzed data. $\mathrm{CA}$ and KW generated the mouse KO model. DMD, KW, and MT supervised experiments and analysis. GB, CA, DMD, KW, CCM, and MT wrote the manuscript. All authors reviewed the manuscript.

\section{Acknowledgments}

This work was supported by NIH R01DC009645 and R01DC012836 to MT and R01DC015052 to CCM, and funding from the National Institute for Health Research (NIHR) Manchester Biomedical Research Centre to CCM.

Address correspondence to: Mustafa Tekin, 1501 NW 10th Avenue, BRB-610 (M-860), University of Miami Miller School of Medicine, Miami, Florida 33136, USA. Phone: 305.243.2381; Email:mtekin@med.miami.edu.
1. Wu DK, Kelley MW. Molecular mechanisms of inner ear development. Cold Spring Harb Perspect Biol. 2012;4(8):a008409.

2. Diaz-Horta O, et al. ROR1 is essential for proper innervation of auditory hair cells and hearing in humans and mice. Proc Natl Acad Sci U S A. 2016;113(21):5993-5998.

3. Whitfield TT. Development of the inner ear. Curr Opin Genet Dev. 2015;32:112-118.

4. Morton CC, Nance WE. Newborn hearing screening - a silent revolution. $N$ Engl J Med. 2006;354(20):2151-2164.

5. Mafong DD, Shin EJ, Lalwani AK. Use of laboratory evaluation and radiologic imaging in the diagnostic evaluation of children with sensorineural hearing loss. Laryngoscope. 2002;112(1):1-7.

6. McClay JE, Booth TN, Parry DA, Johnson R Roland P. Evaluation of pediatric sensorineural hearing loss with magnetic resonance imaging. Arch Otolaryngol Head Neck Surg. 2008;134(9):945-952.

7. Tekin M, et al. Homozygous mutations in fibroblast growth factor 3 are associated with a new form of syndromic deafness characterized by inner ear agenesis, microtia, and microdontia. Am J Hum Genet. 2007;80(2):338-344.

8. Everett LA, et al. Pendred syndrome is caused by mutations in a putative sulphate transporter gene (PDS). Nat Genet. 1997;17(4):411-422.

9. Bademci G, et al. FOXF2 is required for cochlear development in humans and mice. Hum Mol Genet. 2019;28(8):1286-1297.

10. Schrauwen I, et al. De novo variants in GREB1L are associated with non-syndromic inner ear malformations and deafness. Hum Genet. 2018;137(6-7):459-470.

11. Robson MI, Ringel AR, Mundlos S. Regula- tory landscaping: how enhancer-promoter communication is sculpted in 3D. Mol Cell. 2019;74(6):1110-1122.

12. Oza AM, et al. Expert specification of the ACMG/ AMP variant interpretation guidelines for genetic hearing loss. Hum Mutat. 2018;39(11):1593-1613.

13. Silberstein $\mathrm{M}$, et al. A system for exact and approximate genetic linkage analysis of SNP data in large pedigrees. Bioinformatics. 2013;29(2):197-205

14. Matsuoka AJ, et al. Directed differentiation of human embryonic stem cells toward placodederived spiral ganglion-like sensory neurons. Stem Cells Transl Med. 2017;6(3):923-936.

15. Gao T, He B, Liu S, Zhu H, Tan K, Qian J. EnhancerAtlas: a resource for enhancer annotation and analysis in 105 human cell/tissue types. Bioinformatics. 2016;32(23):3543-3551.

16. Wang Y, et al. The 3D Genome Browser: a webbased browser for visualizing 3D genome organization and long-range chromatin interactions. Genome Biol. 2018;19(1):151.

17. Settle SH, Rountree RB, Sinha A, Thacker A, Higgins K, Kingsley DM. Multiple joint and skeletal patterning defects caused by single and double mutations in the mouse Gdf6 and Gdf5 genes. Dev Biol. 2003;254(1):116-130.

18. Indjeian VB, et al. Evolving new skeletal traits by cis-regulatory changes in bone morphogenetic proteins. Cell. 2016;164(1-2):45-56.

19. Wang J, et al. A new subtype of multiple synostoses syndrome is caused by a mutation in GDF6 that decreases its sensitivity to noggin and enhances its potency as a BMP signal. J Bone Miner Res. 2016;31(4):882-889.

20. Asai-Coakwell M, et al. GDF6, a novel locus for a spectrum of ocular developmental anomalies. Am J Hum Genet. 2007;80(2):306-315.
21. Tassabehji M, et al. Mutations in GDF6 are associated with vertebral segmentation defects in Klippel-Feil syndrome. Hum Mutat. 2008;29(8):1017-1027.

22. Terhal PA, et al. Further delineation of the GDF6 related multiple synostoses syndrome. $\mathrm{Am} \mathrm{JMed}$ Genet A. 2018;176(1):225-229.

23. Drage Berentsen R, Haukanes BI, Júlíusson PB, Rosendahl K, Houge G. A novel GDF6 mutation in a family with multiple synostoses syndrome without hearing loss. Mol Syndromol. 2019;9(5):228-234.

24. Pregizer S, Mortlock DP. Control of BMP gene expression by long-range regulatory elements. Cytokine Growth Factor Rev. 2009; 20(5-6):509-515.

25. Ovcharenko I, Loots GG, Nobrega MA, Hardison RC, Miller W, Stubbs L. Evolution and functional classification of vertebrate gene deserts. Genome Res. 2005;15(1):137-145.

26. Osterwalder M, et al. Enhancer redundancy provides phenotypic robustness in mammalian development. Nature. 2018;554(7691):239-243.

27. Anderson E, Devenney PS, Hill RE, Lettice LA. Mapping the Shh long-range regulatory domain. Development. 2014;141(20):3934-3943.

28. Johnson KR, et al. Deletion of a long-range $D l x 5$ enhancer disrupts inner ear development in mice. Genetics. 2018;208(3):1165-1179.

29. Naranjo S, et al. Multiple enhancers located in a 1-Mb region upstream of POU3F 4 promote expression during inner ear development and may be required for hearing. Hum Genet. 2010;128(4):411-419.

30. Rudnicki A, et al. Next-generation sequencing of small RNAs from inner ear sensory epithelium identifies microRNAs and defines regulatory pathways. BMC Genomics. 2014;15:484. 